\title{
DEEP INELASTIC SCATTERING FROM LIGHT NUCLEI
}

\author{
W. MELNITCHOUK ${ }^{1}$, A. W. THOMAS ${ }^{2}$ \\ 1 Jefferson Lab, 12000 Jefferson Avenue, Newport News, VA 23606 \\ 2 Department of Physics and Mathematical Physics, and Centre for the \\ Subatomic Structure of Matter, University of Adelaide, 5005, Australia
}

\begin{abstract}
We review recent developments in the study of deep inelastic scattering from light nuclei, focusing in particular on deuterium, helium, and lithium. Understanding the nuclear effects in these systems is essential for the extraction of information on the neutron structure function.
\end{abstract}

\section{Introduction}

For the past 20 years the nuclear EMC effect - the nuclear medium modification of the nucleon structure functions - has posed a serious challenge to models of the nucleus which involve quark degrees of freedom. Although the basic features of the EMC effect can be understood within a conventional nuclear physics framework, a quantitative description over the entire range of Bjorken- $x$ appears beyond the capacity of a single mechanism. This has led to an assortment of nuclear models and effects which have been postulated to account for the medium modifications of the structure functions.

An impressive array of data on nuclear structure functions has by now been accumulated from experiments at CERN, SLAC, DESY (Hermes), Fermilab, Jefferson Lab and elsewhere, for a broad range of nuclei, and the quality of recent data exploring extreme kinematics, both at low $x$ and high $x$, is pushing nuclear models to their limits. Surprisingly, the EMC effect in the lightest nuclei - in particular the $A=2$ and $A=3$ systems, where microscopic few-body calculations with realistic potentials are more feasible - is still unknown, leaving the determination of the $A$ dependence of the effect incomplete. In addition, the absence of data on the EMC effect in deuterium and helium nuclei prevents the unambiguous determination of the structure function of the free neutron, for which these nuclei are often used as effective neutron targets.

Here we review the foundations of the conventional approach to deep 
inelastic scattering (DIS) from few body nuclei, starting from a covariant, relativistic framework. We focus specifically on the intermediate and large $x$ region dominated by valence quarks, and do not discuss the region of nuclear shadowing and antishadowing at $x<0.1$. As examples, we describe the nuclear effects in spin-averaged and spin-dependent structure functions of the deuteron, the $A=3$ mirror nuclei, and lithium isotopes, paying particular attention to the nuclear corrections which need to be applied when extracting neutron structure functions.

\section{Formalism}

Away from the small- $x$ region $(x>0.2-0.3)$, DIS from a nucleus is computed in the nuclear impulse approximation in terms of incoherent scattering from individual bound nucleons in the nuclear target. ${ }^{1,2}$ The hadronic tensor for a nucleus $A$ can be written as ${ }^{2,3}$

$$
W_{\mu \nu}^{A}(P, q)=\int d^{4} p \operatorname{Tr}\left[\widehat{A}_{N A}(P, p) \cdot \widehat{W}_{\mu \nu}^{N}(p, q)\right],
$$

where $P, p$ and $q$ are the target nucleus, scattered nucleon, and photon momenta, respectively. For illustration we consider the case of spin-averaged DIS; the generalization to spin-dependent scattering is straightforward. ${ }^{4,5,6}$ In this case, the most general expression for the truncated (off-shell) nucleon tensor is given $b^{2}$

$$
\widehat{W}_{\mu \nu}^{N}(p, q)=g_{\mu \nu}\left(I \widehat{W}_{0}+\not p \widehat{W}_{1}+\not q \widehat{W}_{2}\right),
$$

where the bound nucleon "structure functions" $\widehat{W}_{0,1,2}$ are functions of $p$ and $q$, and the (off-shell) nucleon-nucleus amplitude can be written as

$$
\widehat{A}_{N A}(P, p)=\left(I A_{S}+\gamma_{\alpha} A_{V}^{\alpha}\right),
$$

where a sum over residual $A-1$ nuclear states is implicit in the functions $A_{S}$ and $A_{V}^{\alpha}$. The spin-averaged $F_{2}$ structure function of the nucleus is then given by

$$
F_{2}^{A}(x)=\int d^{4} p\left(A_{S} \widehat{W}_{0}+p \cdot A_{V} \widehat{W}_{1}+q \cdot A_{V} \widehat{W}_{2}\right) .
$$

In general the nuclear structure function cannot be written as a one-dimensional convolution integral ${ }^{7}$ even in the impulse approximation: factorization of amplitudes does not imply factorization of structure functions. ${ }^{2}$ However, by taking either selective on-shell ${ }^{2,8}$ or nonrelativistic ${ }^{6,9}$ limits, one can identify a convolution component, plus offshell $\left(p^{2} \neq M^{2}\right)$ corrections,

$$
F_{2}^{A}(x)=\sum_{N} f_{N / A} \otimes F_{2}^{N}(x)+\delta^{(\text {off })} F_{2}^{A}(x),
$$


where $\otimes$ denotes convolution, $f_{N / A} \otimes F_{2}^{N}(x) \equiv \int d y f_{N / A}(y) F_{2}^{N}(x / y)$, with $y=p \cdot q / P \cdot q$ the light-cone fraction of the nucleus carried by the interacting nucleon.

\section{Deuterium}

Being the simplest nuclear system, the deuteron has been studied most extensively, both in non-relativistic and relativistic analyses. Within the formalism described in Section 2, the deuteron $F_{2}$ structure function can be calculated by smearing the nucleon structure functions with the nucleon light-cone momentum distribution in the deuteron, $f_{N / D}(y)$. The off-shell corrections can be evaluated in terms of the relativistic components of the deuteron wave functions and the $p^{2}$ dependence of the truncated nucleon structure functions, ${ }^{8}$ and are typically found to be rather small, $\left|\delta^{(\text {off })} F_{2}^{D}\right| \lesssim$ $1 \%$ for all $x$.
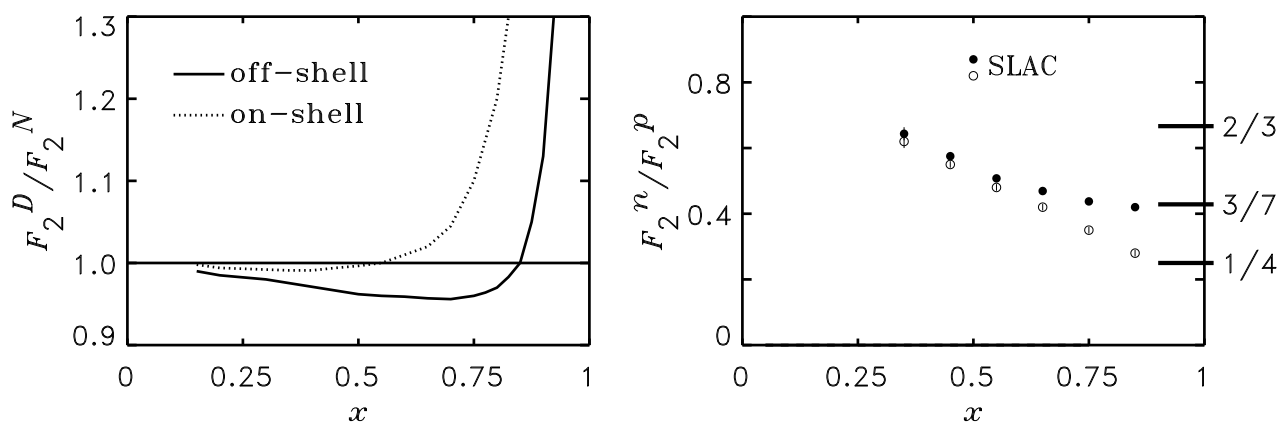

Figure 1. Left panel: ratio of the deuteron to nucleon $F_{2}$ structure functions in the relativistic (off-shell) model $^{8}$ (solid), and an on-shell model ${ }^{10}$ including Fermi motion only. Right panel: extracted neutron to proton ratio using the off-shell and on-shell models of the deuteron. ${ }^{11}$

The calculated $F_{2}^{D}$ structure function for the relativistic model, including the small off-shell correction, is shown in Fig. 1 (left panel) as a ratio to the free nucleon structure function (solid curve). Also shown is the result of an on-shell ansatz ${ }^{10}$ in which (effectively) only Fermi motion effects are included. This gives a much smaller EMC effect in the deuteron. From the measured deuteron and proton structure functions, and the smearing function, $f_{N / D}(y)$, one can extract the structure function of the neutron using the iterative deconvolution procedure ${ }^{12,13}$ (which does not assume 
prior knowledge of $F_{2}^{n}$ ). Using $F_{2}^{p}$ and $F_{2}^{D}$ data from SLAC, ${ }^{14}$ the resulting $F_{2}^{n} / F_{2}^{p}$ ratio is shown in Fig. 1 (right panel) for the relativistic ${ }^{11,15}$ and on-shell models (open and filled circles, respectively). The larger $F_{2}^{n} / F_{2}^{p}$ values in the former reflect a larger EMC effect in the deuteron. Despite the deuteron being a loosely bound system, the nuclear effects at large $x$ can therefore play an important role in the extraction of the free neutron structure function.

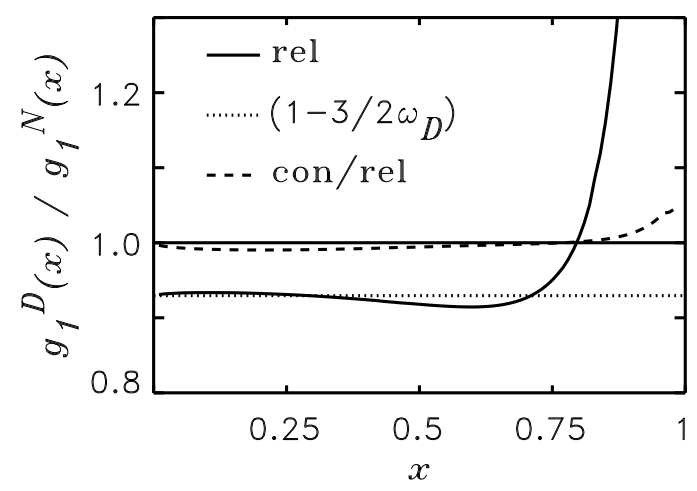

Figure 2. Ratio of deuteron and nucleon $g_{1}$ structure functions in the relativistic model ${ }^{4}$ (solid), and with a constant depolarization factor (dotted). Also shown is the ratio of the convolution component of $g_{1}^{D}$ to the total (dashed).

A similar analysis can be performed for the spin-dependent $g_{1}$ structure function of the deuteron, in terms of the spin-dependent nucleon momentum distribution, $\Delta f_{N / D}(y)$, and relativistic (off-shell) corrections, ${ }^{4}$

$$
g_{1}^{D}(x)=\sum_{N} \Delta f_{N / D} \otimes g_{1}^{N}(x)+\delta^{(\text {off })} g_{1}^{D}(x),
$$

where $\Delta f_{N / D}$ is normalized to $\int d y \Delta f_{N / D}(y)=1-\frac{3}{2} \omega_{D}$, with $\omega_{D}$ the deuteron $D$ state probability. The resulting ratio of the deuteron to nucleon $g_{1}$ structure functions is illustrated in Fig. 2. Also shown is the result assuming $\delta^{\text {(off) }} g_{1}^{D} \rightarrow 0$ and $\Delta f_{N / D}(y) \rightarrow\left(1-\frac{3}{2} \omega_{D}\right) \delta(1-y)$, in which the ratio is given by a constant depolarization factor for all $x$. For $x<0.7$ the nuclear effects are weakly dependent on $x$, so that the use of a constant depolarization factor is a reasonable approximation, although it breaks down dramatically for $x>0.75$. Furthermore, the convolution approximation is quite accurate for $x<0.8$, with $\lesssim 1-2 \%$ deviations from the full result. 
The largest uncertainty in the extraction of $g_{1}^{n}$ over this range of $x$ is due to uncertainty in the $D$-state probability.

\section{4. $A=3$ Nuclei}

As the lightest mirror nuclei, ${ }^{3} \mathrm{He}$ and ${ }^{3} \mathrm{H}$ provide a unique laboratory for studying nuclear effects in structure functions, since many of their properties are similar up to nuclear charge symmetry breaking effects. Furthermore, since the doubly represented nucleons tend to couple to spin 0 , the spin of the $A=3$ nuclei is carried largely by the singly represented nucleon ( $n$ in the case of ${ }^{3} \mathrm{He}, p$ in the case of ${ }^{3} \mathrm{H}$ ).

At present the size of the nuclear EMC effect in ${ }^{3} \mathrm{H}$ is not known, and only scant data exist ${ }^{16}$ on the $F_{2}$ structure function of ${ }^{3} \mathrm{He}$ at lower $Q^{2}$. The predicted nuclear EMC ratio for ${ }^{3} \mathrm{He}$,

$$
R\left({ }^{3} \mathrm{He}\right)=\frac{F_{2}^{3} \mathrm{He}}{2 F_{2}^{p}+F_{2}^{n}},
$$

is plotted in Fig. 3 (left panel), where $F_{2}^{3} \mathrm{He}$ is evaluated as in Eq. (5) via a convolution of the bound $p$ and $n$ structure functions with the nucleon momentum distributions in ${ }^{3} \mathrm{He}, f_{N /{ }^{3} \mathrm{He}}(y)$. The latter are obtained from three-body wave functions calculated by solving the Faddeev equations with a modified Paris potential ${ }^{17}$ (dashed) and via a variational approach ${ }^{18,19}$ (dot-dashed). Since relativistic wave functions of $A=3$ nuclei are not yet available, we cannot quantify the off-shell correction, $\delta^{\text {(off) }} F_{2}^{3} \mathrm{He}$. However, analysis of quasi-elastic scattering from ${ }^{4} \mathrm{He}$, in which binding effects are larger than those in ${ }^{3} \mathrm{He}$, suggests ${ }^{20}$ rather small modifications in the intrinsic nucleon structure $(<5 \%$ for $x<0.8)$. The theoretical ratios are compared with data from HERMES ${ }^{16}$ on the ratio of ${ }^{3} \mathrm{He}$ to $D+p$ structure functions, and agree quite well within the current errors. ${ }^{21}$ The effect on $R\left({ }^{3} \mathrm{He}\right)$ of including the EMC effect in the deuteron (solid) is relatively small for $x<0.8$.

While the model dependence of the EMC effect in ${ }^{3} \mathrm{He}$ is at the few $\%$ level, the fact that ${ }^{3} \mathrm{He}$ and ${ }^{3} \mathrm{H}$ are mirror nuclei means that one can expect similar medium modification in both nuclei. This is illustrated in Fig. 3 (right panel), where the ratio of EMC ratios for ${ }^{3} \mathrm{He}$ and ${ }^{3} \mathrm{H}, \mathbf{R} \equiv$ $R\left({ }^{3} \mathrm{He}\right) / R\left({ }^{3} \mathrm{H}\right)$, is plotted for the standard Faddeev wave function (solid), ${ }^{22}$ and a modified wave function including effects of charge symmetry breaking (dashed) which accounts for the difference in binding energies of ${ }^{3} \mathrm{He}$ and ${ }^{3} \mathrm{H}$. For comparison, the result of the density extrapolation model is also shown. Even though this model ${ }^{10}$ predicts a difference in the absolute size of the EMC effect between ${ }^{3} \mathrm{He}$ and ${ }^{3} \mathrm{H}$ of order $40 \%$, it nevertheless gives 

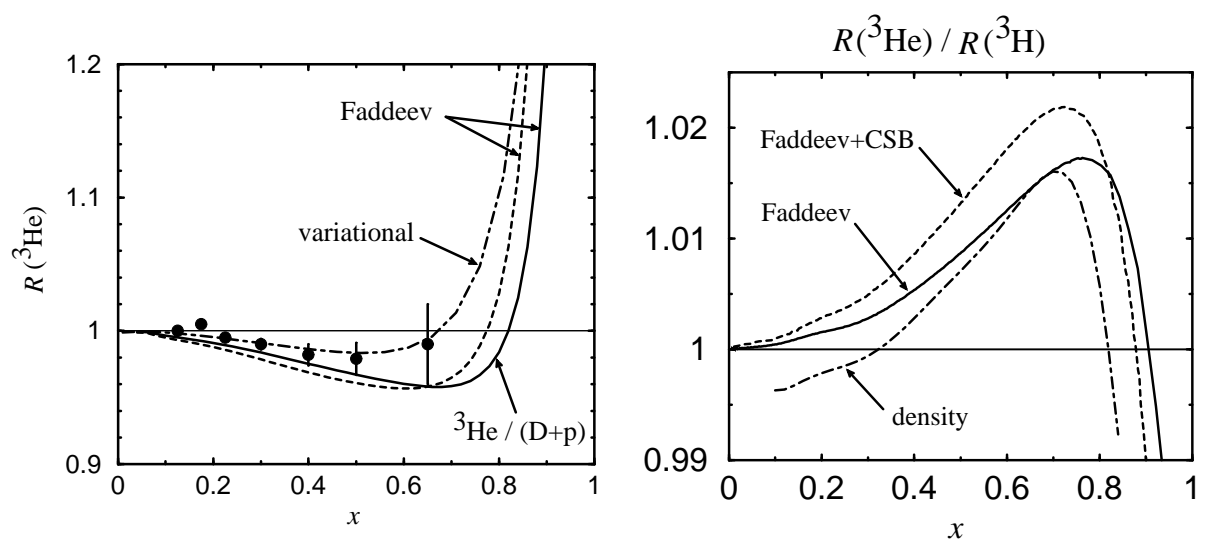

Figure 3. Left panel: nuclear EMC ratio in ${ }^{3} \mathrm{He}$, compared with data on the ${ }^{3} \mathrm{He} /(D+p)$ ratio. ${ }^{16}$ The effect of nuclear corrections in the deuteron is indicated by the solid curve. Right panel: ratio of EMC ratios in ${ }^{3} \mathrm{He}$ and ${ }^{3} \mathrm{H}$, for the Faddeev wave function model (solid), including explicit charge symmetry breaking effects (dashed), ${ }^{22}$ and an ansatz based on the nuclear density extrapolation model. ${ }^{23}$

rise to a similar effect in the ratio $\mathbf{R} \cdot{ }^{23}$ From the calculated ratio $\mathbf{R}$ and a measurement of the ratio of the ${ }^{3} \mathrm{He}$ and ${ }^{3} \mathrm{H}$ structure functions, one can directly extract the neutron to proton ratio, ${ }^{22}$

$$
\frac{F_{2}^{n}}{F_{2}^{p}}=\frac{2 \mathbf{R}-F_{2}^{3} \mathrm{He} / F_{2}^{3} \mathrm{H}}{2 F_{2}^{3} \mathrm{He} / F_{2}^{3} \mathrm{H}-\mathbf{R}}
$$

with significantly less sensitivity to nuclear effects than in Fig. 1 using the inclusive deuteron data. ${ }^{22,24}$

Turning to the spin polarized $A=3$ system, the fact that in ${ }^{3} \mathrm{He}$ the $p p$ pair couples predominantly to spin 0 allows polarized ${ }^{3} \mathrm{He}$ to be used as an effective polarized neutron target ${ }^{25,26}$. The $g_{1}$ structure of ${ }^{3} \mathrm{He}$ can be written as

$$
g_{1}^{3} \mathrm{He}(x) \approx 2 \Delta f_{p /{ }^{3} \mathrm{He}} \otimes g_{1}^{p}(x)+\Delta f_{n /{ }^{3} \mathrm{He}} \otimes g_{1}^{n}(x),
$$

where the spin-dependent nucleon momentum distributions in ${ }^{3} \mathrm{He}$ are normalized such that ${ }^{17,27} \int d y \Delta f_{n /{ }^{3} \mathrm{He}}(y) \equiv \rho_{n} \approx 88 \%$ and $\int d y \Delta f_{p /{ }^{3} \mathrm{He}}(y) \equiv$ $\rho_{p} \approx-2 \%$. The similarity between the ${ }^{3} \mathrm{He}$ and neutron $g_{1}$ structure functions is illustrated in Fig. 4 (left panel), reflecting the small proton contribution to the ${ }^{3} \mathrm{He}$ polarization. For a polarized ${ }^{3} \mathrm{H}$ target, the difference between the $g_{1}$ structure function of ${ }^{3} \mathrm{H}$,

$$
g_{1}^{3} \mathrm{H}(x) \approx \Delta f_{p /{ }^{3} \mathrm{H}} \otimes g_{1}^{p}(x),
$$

and $g_{1}^{p}$ would be even smaller because, in addition to the small size of the neutron polarization in ${ }^{3} \overrightarrow{\mathrm{H}}$, the intrinsic magnitude of $g_{1}^{n}$ is much smaller 
than that of $g_{1}^{p}$. A measurement of $g_{1}^{3} \mathrm{H}$ would therefore allow an accurate determination of the $\Delta f_{p /{ }^{3} \mathrm{H}} \approx \Delta f_{n /{ }^{3} \mathrm{He}}$ distribution, which could then be used to minimize the uncertainty in the extraction of $g_{1}^{n}$ from $g_{1}^{3} \mathrm{He}$. The effects of possible $\Delta$ resonance components in the ${ }^{3} \mathrm{He}$ wave function have also been examined by Bissey et al. ${ }^{28}$
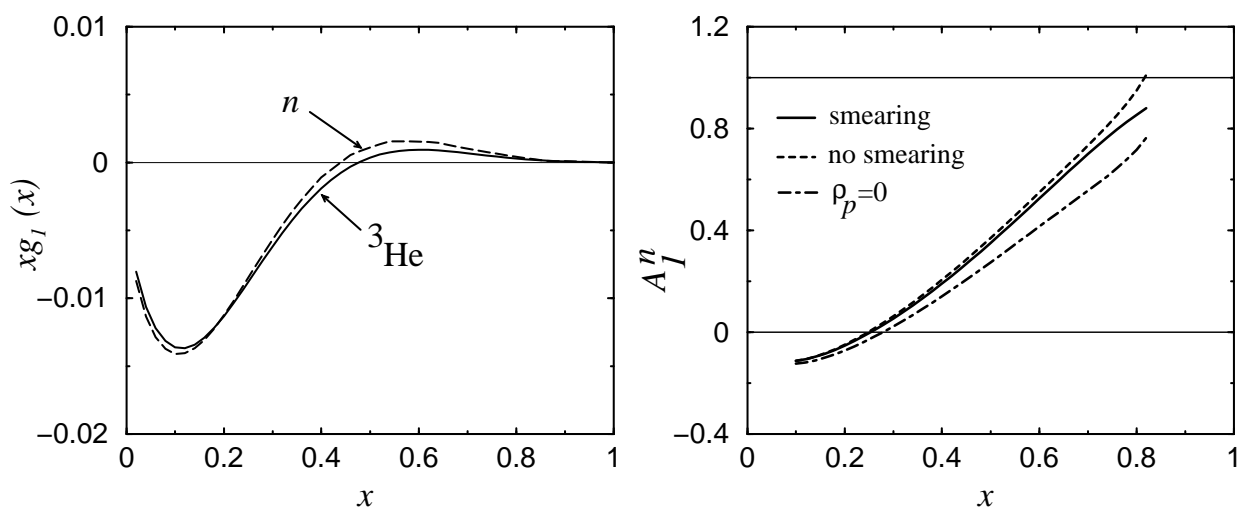

Figure 4. Left panel: $x g_{1}$ structure function of the neutron and ${ }^{3} \mathrm{He}$. Right panel: neutron polarization asymmetry $A_{1}^{n}$ obtained from the ${ }^{3} \mathrm{He}$ asymmetry including smearing corrections (solid), without smearing (dashed), and neglecting the proton polarization, $\rho_{p}=0$ (dot-dashed).

Because the $g_{1}^{n}$ structure function changes sign at intermediate $x$, it is not meaningful to calculate an EMC-type ratio. On the other hand, taking the ratio of the $g_{1}$ to $F_{1}$ structure functions (where $F_{1} \approx F_{2} / 2 x$ at large $Q^{2}$ ), one can better visualize the nuclear corrections in the polarization asymmetry,

$$
A_{1}(x) \approx \frac{g_{1}(x)}{F_{1}(x)},
$$

which is the quantity measured directly in polarized DIS experiments. In Fig. 4 (right panel) the $A_{1}^{n}$ asymmetry is shown, extracted from the ${ }^{3} \mathrm{He}$ asymmetry including smearing corrections (solid). For comparison, the result without smearing is also shown (dashed) - in this case the distributions $\Delta f_{N /{ }^{3} \mathrm{He}}(y) \rightarrow \rho_{N} \delta(1-y)$, and the shapes of $g_{1}^{3} \mathrm{He}$ and $g_{1}^{n}$ are assumed to be the same. This appears to be a reasonable approximation for $x<0.7$, but breaks down beyond $x \sim 0.8$. Over the same region of $x$ the proton polarization is also seen to play a relatively minor role, although it must be included if precision at the level of a few percent is required. 
An accurate determination of nuclear effects is also necessary for the extraction of the $g_{2}$ structure function of the neutron. The $g_{2}$ structure function provides the cleanest means to access information on higher twist contributions in polarized DIS. Of particular importance is the $x^{2}$-weighted moment of $g_{2}$, which enters in the twist- $3 d_{2}$ matrix element. The nuclear corrections to the neutron $g_{2}$ structure function are illustrated in Fig. 5, where the $x^{2}$-weighting emphasizes the larger- $x$ region. Since there is practically no information on the twist-3 component of $g_{2}$, the calculation has been performed assuming twist-2 dominance of $g_{2}^{n}$. The effect of smearing appears to be small, although not negligible, and needs to be included for an accurate determination of $g_{2}^{n}$ at large $x$.

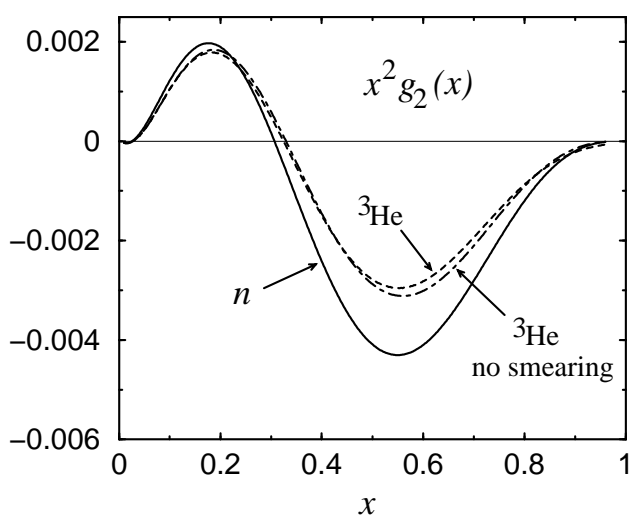

Figure 5. $\quad x^{2}$-weighted $g_{2}$ structure function of the neutron (solid), and of ${ }^{3} \mathrm{He}$, including smearing corrections (dashed), and without smearing (dot-dashed).

\section{Lithium}

$\beta$-unstable $\mathrm{Li}$ isotopes provide a promising new avenue for exploring the quark structure of few body nuclei. The ${ }^{11} \mathrm{Li}$ nucleus, which is located at the neutron drip line, is believed to be well approximated as a ${ }^{9} \mathrm{Li}$ core plus two weakly bound valence neutrons, which form a halo structure. Because of the weak binding, it has been suggested ${ }^{29}$ that the neutron momentum distribution in ${ }^{11} \mathrm{Li}$ should be very sharp and symmetric around $y=1$, making it an excellent candidate for an effective neutron target. Polarized ${ }^{6} \mathrm{Li}$ nuclei, which have with spin 1 and isospin 0 , have been suggested as effective polarized $(\approx 86 \%$ ) deuteron targets, in the approximation that ${ }^{6} \overrightarrow{\mathrm{Li}} \approx \alpha+\vec{D}$. Furthermore, polarized ${ }^{7,9,11} \mathrm{Li}$ isotopes can also be used to study the novel structure function ${ }^{29,30}$ associated with spin $3 / 2$ systems, 
${ }_{3}^{3 / 2} g_{1}$. Deep inelastic scattering from the ${ }^{7} \mathrm{Li}^{-}{ }^{7} \mathrm{Be}$ mirror nuclei can also be used to explore the medium modifications of the Bjorken and Gottfried sum rules. ${ }^{29,31}$ Studies with these nuclei may one day be undertaken at radioactive beam facilities, such as those proposed at RIKEN or GSI. The merits of using other nuclear targets, such as nitrogen, have been elaborated by Rondon. ${ }^{32}$

\section{Conclusions}

The unprecedented quality of recent data on few body nuclear structure functions from new generations of accelerators is demanding better understanding of nuclei in extreme kinematic regions, such as at large $x$, where the effects of relativity and nucleon substructure play a more prominent role. This challenge is being met by concurrent progress being made in the theory of DIS from few body nuclei, especially deuterium and $A=3$ nuclei.

In addition to more accurately determining the response of these nuclei to electromagnetic probes, an important practical necessity lies in controlling the nuclear corrections when extracting information on the structure of the neutron, for which light nuclei are often used as effective neutron targets. While much of the focus has been on the $F_{2}$ structure function, there is a growing need to understand the nuclear effects in other structure functions, such as $g_{1}$ and $g_{2}$, as well as the new structure functions available for higher spin nuclei — both in the deep inelastic and resonance regions. The interplay between theoretical developments and anticipated future data promises to provide even deeper insights into the quark structure of nuclei.

\section{Acknowledgments}

We are grateful to F. Bissey and S. Liuti for providing their light-cone momentum distribution functions. This work was supported by the U.S. Department of Energy contract DE-AC05-84ER40150, under which the Southeastern Universities Research Association (SURA) operates the Thomas Jefferson National Accelerator Facility (Jefferson Lab) and by the Australian Research Council. 


\section{References}

1. D. F. Geesaman, K. Saito and A. W. Thomas, Ann. Rev. Nucl. Part. Sci. 45, 337 (1995).

2. W. Melnitchouk, A. W. Schreiber and A. W. Thomas, Phys. Rev. D 49, 1183 (1994), nucl-th/9311008.

3. F. Gross and S. Liuti, Phys. Rev. C 45, 1374 (1992).

4. W. Melnitchouk, G. Piller and A. W. Thomas, Phys. Lett. B 346, 165 (1995), hep-ph/9501282.

5. G. Piller, W. Melnitchouk and A. W. Thomas, Phys. Rev. C 54, 894 (1996), nucl-th/9605045.

6. S. A. Kulagin, W. Melnitchouk, G. Piller and W. Weise, Phys. Rev. C 52, 932 (1995), hep-ph/9504377.

7. R. L. Jaffe, in Relativistic Dynamics and Quark-Nuclear Physics, M. B. Johnson and A. Pickleseimer, eds. (Wiley, New York, 1985).

8. W. Melnitchouk, A. W. Schreiber and A. W. Thomas, Phys. Lett. B 335, 11 (1994), nucl-th/9407007.

9. S. A. Kulagin, G. Piller and W. Weise, Phys. Rev. C 50, 1154 (1994), nuclth/9402015.

10. L. L. Frankfurt and M. I. Strikman, Phys. Rep. 76, 215 (1981); ibid 160, 235 (1988).

11. W. Melnitchouk and A. W. Thomas, Phys. Lett. B 377, 11 (1996), nuclth/9602038.

12. A. Bodek et al., Phys. Rev. D 20, 1471 (1979).

13. W. Melnitchouk and A. W. Thomas, Acta Phys. Polon. B 27, 1407 (1996), nucl-th/9603021.

14. L. W. Whitlow et al., Phys. Lett. B 282, 475 (1992).

15. A. W. Thomas and W. Melnitchouk, Nucl. Phys. A631, 296C (1998), hep$\mathrm{ph} / 9708484$.

16. K. Ackerstaff et al., Phys. Lett. B 475, 386 (2000).

17. F. Bissey, A. W. Thomas and I. R. Afnan, Phys. Rev. C 64, 024004 (2001), nucl-th/0012081; A. W. Thomas, I. R. Afnan and F. Bissey, RIKEN Rev. 28, 90 (2000).

18. C. Ciofi degli Atti, E. Pace and G. Salme, Phys. Rev. C 21, 805 (1980); Phys. Lett. 141 B, 14 (1984).

19. C. Ciofi degli Atti and L. Liuti, Phys. Rev. C 41, 1100 (1990); ibid C 44, 1269 (1991).

20. W. Melnitchouk, K. Tsushima and A. W. Thomas, Eur. Phys. J. A 14, 105 (2002), nucl-th/0110071.

21. F. Bissey et al., in preparation.

22. I. R. Afnan, F. Bissey, J. Gomez, A. T. Katramatou, W. Melnitchouk, G. G. Petratos and A. W. Thomas, Phys. Lett. B 493, 36 (2000), nuclth/0006003; G. G. Petratos, et al., nucl-ex/0010011.

23. W. Melnitchouk, I. R. Afnan, F. Bissey and A. W. Thomas, Phys. Rev. Lett. 84, 5455 (2000), hep-ex/9912001.

24. E. Pace, G. Salme, S. Scopetta and A. Kievsky, Phys. Rev. C 64, 055203 (2001), nucl-th/0109005; M. M. Sargsian et al., nucl-th/0105052.

25. B. Blankleider and R. M. Woloshyn, Phys. Rev. C 29, 538 (1984). 
26. J. L. Friar et al., Phys. Rev. C 42, 2310 (1990).

27. C. Ciofi degli Atti, S. Scopetta, E. Pace and G. Salme, Phys. Rev. C 48, 968 (1993), nucl-th/9303016;

28. F. Bissey et al., Phys. Rev. C 65, 064317 (2002), hep-ph/0109069.

29. K. Saito, M. Ueda, K. Tsushima and A. W. Thomas, Nucl. Phys. A705, 119 (2002), nucl-th/0110024.

30. R. L. Jaffe and A. Manohar, Nucl. Phys. B321, 343 (1989).

31. V. Guzey and M. Strikman, Phys. Rev. C 61, 014002 (2000).

32. O. A. Rondon, Phys. Rev. C 60, 035201 (1999). 